\title{
Less may be more: Using small molecules to reprogram human cells into functional cardiomyocytes
}

\author{
Prashant S. Kota, BS, Mostafa R. Naguib, Vivekkumar Patel, MD, and Todd K. Rosengart, MD, FACS
}

\begin{abstract}
The prospect of genetically reprogramming cardiac fibroblasts into induced cardiomyocytes by using cardio-differentiating transcription factors represents a significant advantage over previous strategies involving stem cell implantation or the delivery of angiogenic factors. Remarkably, intramyocardial administration of cardio-differentiating factors consistently results in $20 \%$ to $30 \%$ improvements in postinfarct ejection fraction and nearly a $50 \%$ reduction in myocardial fibrosis in murine models. Despite these encouraging observations, few breakthroughs have been made in the reprogramming of human cells, which have more rigorous epigenetic constraints and gene regulatory networks that oppose reprogramming. As a potential solution to this challenge, Cao and colleagues used a cocktail of 9 chemicals capable of reprogramming human fibroblasts into contractile cardiomyocyte-like cells, albeit at a low efficiency. This strategy would obviate the concerns with viral vectors and appears to partially overcome the epigenetic constraints in human cells. Nevertheless, significant challenges, including drug-drug interactions, low reprogramming efficiency, and lack of in vivo data must be overcome before future clinical application. (J Thorac Cardiovasc Surg 2017;153:128-30)
\end{abstract}

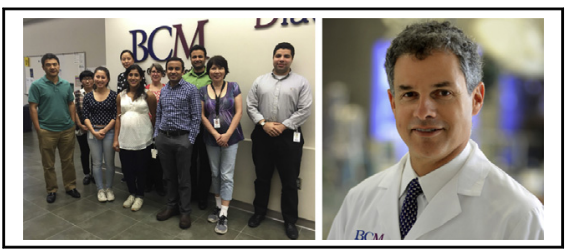

Todd K. Rosengart, MD, FACS

\section{Central Message}

Small molecule mediated reprogramming of human fibroblasts into functional cardiomyocytelike cells may represent a promising therapeutic option for cardiac regeneration.

See Editorial Commentary page 130.
Feature Editor's Note-Readers who remember being burned by the specious promise of laser transmyocardial revascularization, which shape-shifted into injection of scarred myocardium with angiogenesis factors, followed by the hope-springs-eternal promises of stem cells and the genome, cannot miss being stimulated by the recent report from Cao and colleagues in Science. ${ }^{1}$ Is it possible that throwing 9 compounds into a blender with some mouse fibroblasts transforms them into "cardiomyocyte-like" cells that display important elements of rudimentary functionality when transplanted into mouse hearts? If this approach does not turn out to be transmyocardial revascularization 4.0, it will be very exciting, and potentially transformative. Regeneration of scarred myocardium might actually be possible on a practical scale. See what Kota, Naguib, Patel, and Rosengart have to say on the topic in their Invited Expert Opinion.

Craig R. Smith, MD

From the Michael E. DeBakey Department of Surgery, Baylor College of Medicine, Houston, Tex.

Received for publication July 13, 2016; revisions received July 30, 2016; accepted for publication Aug 1, 2016; available ahead of print Oct 7, 2016

Address for reprints: Todd K. Rosengart, MD, FACS, Michael E. DeBakey Department of Surgery, 6501 Fannin St, Ste NC114, Houston, TX 77030 (E-mail: Todd.Rosengart@bcm.edu).

$0022-5223 / \$ 36.00$

Copyright $(2) 2016$ by The American Association for Thoracic Surgery

http://dx.doi.org/10.1016/j.jtcvs.2016.08.039
Congestive heart failure is among the leading causes of morbidity and mortality in the United States. Despite many advances, current treatments for patients with end-stage congestive heart failure involving heart organ transplants and mechanical circulatory support are still limited by the availability of organ donors and the morbidity and mortality associated with these procedures. However, a new era of heart failure treatment may be approaching, ushered in by the 2010 demonstration by Ieda and colleagues ${ }^{2}$ that cardiac fibroblasts could be genetically reprogrammed into induced cardiomyocytes (iCMs), and that this cellular transdifferentiation strategy could be used to improve postinfarct ventricular function in vivo. ${ }^{2}$

Building on Yamanaka's Nobel Prize-winning discovery that induced pluripotent stem cells (iPSs) could be generated from adult somatic cells, the novel approach of "direct cellular reprogramming" first reported by Ieda and colleagues ${ }^{2}$ uses cardio-differentiating factors that bypass iPS staging in generating cardiomyocyte-like cells directly from what has perennially been presumed to be terminally differentiated scar fibroblasts. This approach avoids potential tumorigenicity and immunogenicity risks associated with iPS cells. Perhaps more importantly, by transdifferentiating infarct fibroblasts in situ, direct cellular reprogramming avoids the many challenges that have long plagued exogenous stem cell delivery strategies for treating heart failure. ${ }^{2}$ 


$$
\begin{aligned}
& \text { Abbreviations and Acronyms } \\
& \begin{aligned}
9 \mathrm{C} & =9 \text { chemicals } \\
\mathrm{CiCM} & =\text { chemically induced cardiomyocyte } \\
\mathrm{iCM} & =\text { induced cardiomyocyte } \\
\mathrm{iPS} & =\text { induced pluripotent stem cell }
\end{aligned}
\end{aligned}
$$

We and others have demonstrated that cellular reprogramming utilizing intramyocardial injection of gene transfer vectors encoding key cardio-differentiating transcription factors such as Gata4, Mef2c, and Tbx5 in rodent models can produce up to $20 \%$ to $30 \%$ increases in postinfarct ejection fraction and nearly $50 \%$ decreases in myocardial fibrosis. ${ }^{3}$ As an alternate to these gene delivery-based strategies, Cao and colleagues ${ }^{1}$ now report that a cocktail of "small molecules" can also be used to induce cardiac transdifferentiation. This innovation may prove to be particularly important given the recent discovery that human cells appear to be more resistant to cellular reprogramming than rodent cells, likely due to the more complex gene regulatory network and epigenetic constraints found in higher-order species. ${ }^{4}$ To overcome this resistance, investigators have had to resort to the use of increasingly complex reprogramming cocktails, and the work by $\mathrm{Cao}$ and colleagues ${ }^{1}$ may represent a simpler route to overcoming this barrier to human cellular reprogramming.

In their recent report, $\mathrm{Cao}$ and colleagues ${ }^{1}$ employed a cocktail of 9 chemicals (9C) that normally regulate cell proliferation, differentiation, chromatin structure, and various other pathways to transdifferentiate human foreskin and lung fibroblasts within 4 weeks into contractile (chemically induced) cardiomyocytes (ciCMs). ${ }^{1}$ Approximately $7 \%$ of such ciCMs expressed the cardiomyocyte markers cardiac troponin $\mathrm{T}$ and demonstrated intracellular sarcomeric structures. Cao and colleagues ${ }^{1}$ reported that these ciCMs closely resembled iPS-derived cardiomyocytes in terms of sarcomeric organization, electrophysiological properties, and transcriptional signature. Remarkably, the generation of beating iCMs reported by $\mathrm{Cao}$ and colleagues ${ }^{1}$ occurred twice as quickly as previous studies' utilized viral factors. ${ }^{1,4}$

Collectively, these observations suggest that chemical cellular reprogramming, especially if reprogramming cocktails can be reduced from the current requirement of 9 constituents, offers the potential advantages of efficient pharmacokinetic delivery to target cells, precise temporal control of cell exposure to reprogramming factors, and the absence of the various risk concerns often associated with gene transfer strategies. ${ }^{4}$ Further, the observation that the 9C cocktail utilized by $\mathrm{Cao}$ and colleagues ${ }^{1}$ was able to induce an "open chromatin" state, resulting in de-repression of the gene activation loci for the key cardio-differentiating factors Gata4, Nkx2.5, Brachury, and Mesp1, points to a pathway of potentially critical importance to human cellular reprogramming.

\section{FUTURE DIRECTIONS}

Although the premise of chemically induced cellular reprogramming highlighted by $\mathrm{Cao}$ and colleagues ${ }^{1}$ is intriguing and potentially pace setting, this approach still faces significant challenges. Clearly, the delivery of 9 separate drugs into infarcted myocardium may not be significantly easier than the delivery of transgenes, nor will the innumerable drug-drug interactions of complex cocktails be readily transferable to human applications. Further, the 7\% transdifferentiation efficiency achieved by the $9 \mathrm{C}$ cocktail is still extremely low, and far below the $20 \%$ to $30 \%$ rate reported by some with the use of viral vectors. As a consequence, it is unclear if this strategy will yield the same level of clinically meaningful results when ultimately tested in vivo, as has already been achieved with gene transfer-based strategies. Likewise, until in vivo testing is undertaken, it is unclear if ciCMs will be sufficiently mature to enhance ventricular contractile function.

Apart from the contribution to improved postinfarct contractile function rendered by the administration of Gata4, Mef2c, and Tbx5 and other cardio-differentiating factors, these factors have also produced as yet unexplained reductions in postinfarct fibrosis. It is possible that a chemical-based reprogramming strategy might unintentionally bypass possibly promiscuous effects of gene-based strategies that may be enhancing cardiac function and reducing fibrosis via mechanisms unrelated specifically to iCM generation. The deployment of chemical reprogramming strategies may well shed light on this question.

Despite the inevitable new questions generated by a potentially major new step forward, the prospect of chemically induced cellular reprogramming represents an exciting new chapter in the rapidly evolving story of cellular reprogramming. Our understanding of this new bioengineering strategy has significantly increased in a short 6 years, and prospects for further novel steps forward in improving these strategies as a precursor to human studies seems brighter by the day. The work by $\mathrm{Cao}$ and colleagues ${ }^{1}$ is a potentially important step forward in this direction.

\section{Conflict of Interest Statement}

Authors have nothing to disclose with regard to commercial support.

The authors thank Dr Jianchang Yang for providing valuable input and suggestions. 


\section{References}

1. Cao N, Huang Y, Zheng J, Spencer CI, Zhang Y, Fu J, et al. Conversion of human fibroblasts into functional cardiomyocytes by small molecules. Science. 2016;352: 1213-20.

2. Ieda M, Fu JD, Delgado-Olguin P, Vedantham V, Hayashi Y, Bruneau BG, et al. Direct reprogramming of fibroblasts into functional cardiomyocytes by defined factors. Cell. 2010;3:375-86.

3. Mathison M, Gersch RP, Nasser A, Lilo S, Korman M, Fourman M, et al. In vivo cardiac cellular reprogramming efficacy is enhanced by angiogenic preconditioning of the infarcted myocardium with vascular endothelial growth factor. $J \mathrm{Am}$ Heart Assoc. 2012;1:e005652.
4. Sadahiro T, Yamanaka S, Ieda M. Direct cardiac reprogramming: progress and challenges in basic biology and clinical applications. Circ Res. 2015;116: 1378-91.

5. He WJ, Hou Q, Han QW, Han WD, Fu XB. Pluripotent reprogramming and lineage reprogramming: promises and challenges in cardiovascular regeneration. Tissue Eng Part B Rev. 2013;20:304-13.

Key Words: reprogramming, transdifferentiation, induced cardiomyocyte, heart failure

\section{EDITORIAL COMMENTARY}

\section{Transdifferentiation: A new frontier in cardiovascular cell therapy}

\author{
Michael Ibrahim, MD, PhD, and Pavan Atluri, MD \\ From the Division of Cardiovascular Surgery, Department of Surgery, University of Pennsylvania, Philadelphia, Pa. \\ Disclosures: Authors have nothing to disclose with regard to commercial support. \\ Received for publication Sept 8, 2016; accepted for publication Sept 9, 2016; available ahead of print Oct 7, 2016. \\ Address for reprints: Pavan Atluri, MD, Division of Cardiovascular Surgery, Department of Surgery, University of \\ Pennsylvania, Silverstein 6, 3400 Spruce St, Philadelphia, PA 19104 (E-mail: pavan.atluri@uphs.upenn.edu). \\ J Thorac Cardiovasc Surg 2017;153:130-1 \\ $0022-5223 / \$ 36.00$ \\ Copyright (C) 2016 by The American Association for Thoracic Surgery \\ http://dx.doi.org/10.1016/j.jtcvs.2016.09.007
}

Heart failure, the final common pathway of cardiac pathologic remodeling, represents the clinical manifestation of loss of cardiomyocyte number and function in a welldescribed downward spiral, where increasing mechanical overload leads to pathologic hypertrophy and progressive cell death. ${ }^{1}$ The holy grail of stem cell therapy has been to replace those cardiomyocytes lost or damaged in the pathogenesis of heart failure. This requires (1) the development of de novo mature contractile cardiomyocytes that are not immunogenic, (2) the engraftment and survival of these cells such that they can make a meaningful contribution to contraction, and (3) electrical coupling of these cells for concerted action. These have proved elusive goals, and an ideal stem cell population has not been identified. The prospect of using transdifferentiation to manipulate native cell populations into viable contractile myocardium represents a potential breakthrough to these problems, as Kota and colleagues discuss in this issue of the Journal. ${ }^{2}$

A decade ago, Takahashi and Yamanaka ${ }^{3}$ showed that it was possible to use a cocktail of cellular reprogramming factors to revert terminally differentiated cells to a state of pluripotency, so-called induced pluripotent stem cells.

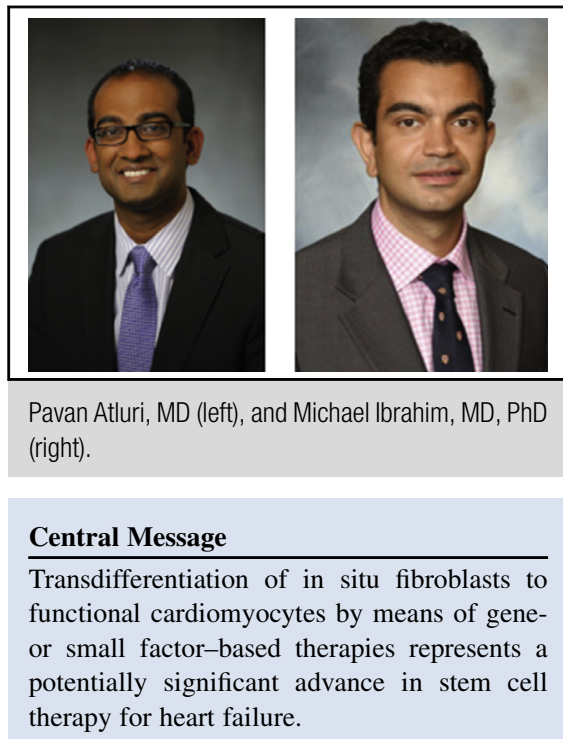

See Article page 128.

These cells may be subsequently directed into cardiomyocyte cell fates, but their use is plagued by many issues, not least by the hurdle of achieving cell engraftment. This problem stimulated the development of direct cellular reprogramming, the use of signaling factors to convert one terminally differentiated cell type into another, without the pluripotent stage.

Fibroblasts, the most numerous cells in the mammalian heart, are a sophisticated population of pleiotropic cells that play a role in scaffolding contracting cardiomyocytes, intercellular signaling, and contraction. ${ }^{4,5}$ Ieda and 